\title{
Specialized drug liaison midwife services for pregnant opioid dependent women in Dublin, Ireland
}

\author{
Mike Scully $^{\mathrm{a}, *}$, Noreen Geoghegan ${ }^{\mathrm{b}}$, Paul Corcoran ${ }^{\mathrm{c}}$, Marika Tiernan $^{\mathrm{b}}$, Eamon Keenan ${ }^{\mathrm{b}}$

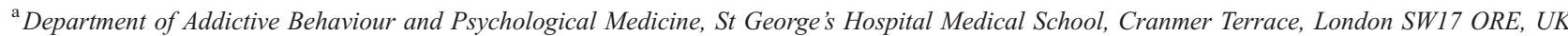 \\ ${ }^{\mathrm{b}}$ Drugs/AIDS Service, South Western Area Health Board, Bridge House, Cherry Orchard Hospital, Ballyfermot, Dublin 10, Ireland \\ ${ }^{\mathrm{c}}$ National Suicide Research Foundation, 1 Perrott Avenue, College Road, Cork, Ireland
}

Received 22 November 2002; received in revised form 2 June 2003; accepted 22 August 2003

\begin{abstract}
The health needs of pregnant opioid dependent women are increasingly being recognized by health care professionals. These women generally receive limited antenatal care. Maternal and neonatal outcomes are also poorer compared to non-drug using women. The number of pregnant opioid dependent women accessing drug treatment services in the Irish Republic has increased. A specialist Drug Liaison Midwife service was created in March 1999 to liaise between the three Dublin Maternity hospitals and the Drug Treatment Services. This paper surveys the first year of operation of one of these posts. It documents sociodemographic background, substance use, and medical histories of these women in addition to maternal and neonatal outcomes. Higher maternal methadone dose was associated with an increased risk of neonatal withdrawals among these women. The experience of this specialist liaison service indicates that it is possible to build effective working relationships between opioid dependent pregnant women and the Obstetric and Drug services involved in their care. This has resulted in benefit to these women, their children and the Irish Health Care system. (C) 2004 Elsevier Inc. All rights reserved.
\end{abstract}

Keywords: Opioid dependent pregnant women; Drug Liaison Midwives; Maternal methadone dose; Neonatal withdrawals

\section{Introduction}

There has been an increasing recognition of the needs of pregnant drug dependent women among professionals involved in their care (Ebrahim \& Gfroerer, 2003; Fischer, 2000). A recent report from the European Monitoring Centre for Drugs and Drug Addiction (2000) has recommended that their care be prioritized. The opioid using population in the Irish Republic and those accessing treatment are younger than other opioid dependent European populations (Department of Tourism, Sport \& Recreation, 2001). The number of dependent pregnant users accessing treatment has increased (Moran, O'Brien, \& Duff, 1997; O'Brien, Moran, Kelleher, \& Cahill, 2000). There has also been an increase in the numbers of infants admitted to Special Care Baby Units with a diagnosis of Neonatal Abstinence Syndrome with obvious bed occupancy and financial implications. This situation was the background

\footnotetext{
* Corresponding author. Tel.: +44 7976232520.

E-mail address: mpscully75@aol.com (M. Scully).
}

to the appointment of Drug Liaison Midwives (DLM) to the three Dublin maternity hospitals in March 1999.

Here we describe an analysis of the referrals to one of these posts, attached to the Coombe Women's Hospital $(\mathrm{CWH})$, a university teaching hospital located in the south inner city area of Dublin that mainly serves patients from the South Western Area Health Board.

\subsection{Methadone treatment}

The oral substitution agent methadone has been licensed for the treatment of opioid dependence since the late 1960s. It was, however, the early part of the 1990s that saw considerable expansion of treatment services with the adoption of a Harm Reduction approach by the Irish government. An account of the development and adoption of Harm Reduction in an Irish context has recently been outlined by Barry (2002).

Oral substitution treatment is now more widely available. The mainstay of treatment is oral methadone. All patients commenced on methadone are registered on a Central Treatment List and all have a designated treatment card 
lodged with their dispensing outlet. All methadone treatment is free.

An innovative shared care approach exists between specialist psychiatric substance misuse services and general practice (Keenan \& Barry, 1999). In general practice settings, only specially trained general practitioners may prescribe methadone for the treatment of opioid dependence. These "level 1 doctors" are limited to prescribing for a limited number of patients who are already stable on oral methadone while level 2 doctors may both initiate methadone stabilization and continue to prescribe for a larger number of patients on an extended basis. Each Health Board serves a defined geographic catchment area. Each Health Board has a specialist GP who coordinates general medical input into the community treatment services with the local Consultant Psychiatrist in Substance Misuse and the local Liaison Pharmacist.

\subsection{Maternal care for opiate-dependent pregnant women}

In Dublin, access to treatment was prioritized for pregnant opioid dependent women. The priority was designed to engage the woman, to have her access both prenatal and drug treatment services quickly, stabilize on oral methadone therapy, and address other health and social care issues as necessary. Stabilization of drug use was stressed and women were encouraged to remain on oral methadone throughout their pregnancy. Dose reduction or escalation was determined by the prescribing doctor in collaboration with the woman. Methadone doses were individualized to take account of individual differences in tolerance and other medical issues (e.g. other medications, HIV, or HCV status). Informed consent to treatment was always sought and the women were aware of their current dose of methadone at all times.

Stability of maternal drug use and engagement with obstetric services were the major treatment priorities. The option to detoxify as an inpatient or outpatient after the first trimester existed, but women were not pressured to reduce dose or to detoxify. Those who had difficulties stabilizing were offered inpatient admission to a specialist drug dependency unit to facilitate stabilization.

After an initial assessment by the DLM, those women not in treatment were immediately offered a treatment place depending on their needs as assessed at the interview with the DLM. All cases were discussed with the Consultant Psychiatrist in Substance Misuse from the relevant Sector team. The settings available for drug treatment included a tertiary treatment center, local methadone treatment centers, and local GP practices where methadone dispensing occurred in a local community pharmacy. All women had prenatal booking appointments made with the obstetric services. Some of these appointments were routine but the facility accelerated appointments in cases where there existed a clinical indication.

A considerable amount of time was spent by the DLM in building working relationships with the women. Issues around confidentiality, contact with social services, etc., were explicitly dealt with and written permission was obtained to authorize contact and disclosure of relevant information. All the women signed a release of information form authorizing the DLM to liaise between the services at the CWH and the local Drugs service.

The current study aimed to document the numbers of women using this new specialist service, and to gain knowledge of their sociodemographic backgrounds and substance use histories. We wanted to document the outcomes for both the mothers and their infants and to look at factors associated with good outcomes.

\section{Methods}

The survey was retrospective and chart based. The Ethics Committee at the $\mathrm{CWH}$ approved the research proposal. Information was gathered from the files held by the DLM using a questionnaire designed for the study. Obstetric and neonatal data were obtained from the patient files held by the Coombe Women's Hospital. Additional data were gathered from the patient files held by the Drugs service. Each pregnancy was counted as a separate case for the purpose of the analysis. Statistical analysis was undertaken using SPSS version 10 .

\section{Results}

In the period from April 1999 to the end of April 2000 there were 111 referrals to the DLM. Two women became pregnant twice during the study period and one woman had twins. Percentages are presented in adjusted form (valid percent) to take account of missing data.

\subsection{Socio-demographic background}

The mean age of this cohort was 23.8 years (range 17-32 years). The majority were unemployed. The mean age of leaving full time education was 15.7 years (range $12-18$ years). Thirty-nine women $(43.8 \%)$ had one state exam, although only $17(19.1 \%)$ had completed the final state exam. The majority were in relationships and 51 $(48.1 \%)$ had drug-using partners. Forty-nine women $(47.6 \%)$ lived with a partner, 27 women $(26.2 \%)$ lived with their families and eight women $(7.8 \%)$ described themselves as homeless.

The mean number of previous pregnancies was 2.21 (range 0-7). The mean number of live births for the sample was 0.9 (range $0-4$ births). Fifty-four women had never given birth previously (48.6\%), 29 women had one child (26.1\%), 15 had two children and only 13 women had three or more children. Only 21 women $(20.8 \%)$ reported that the pregnancy was planned. Only 15 women reported using regular contraception. The majority of these 
women were using condoms, with two using Depo-Provera (medroxyprogestrone acetate - a long acting parenteral progestogen-only contraceptive).

\subsection{Drug history}

Eighty-three women were in substance abuse treatment at the time of referral to the DLM. Their mean age of first using heroin was 18.3 years (range $9-32$ years). Their mean age of first injecting heroin was 19.8 years (range 15-30 years) and the mean duration of injecting heroin use was 20.61 months (range $0-108$ months) (Table 1 ). A history of polysubstance use was common. Only three women did not use tobacco while $34(35.8 \%)$ used alcohol. Fifty-six women $(73.7 \%)$ gave histories of using benzodiazepines, 40 women $(52.6 \%)$ of using cocaine, and 10 women $(22.7 \%)$ of using tricyclic anti-depressant drugs.

\subsection{Forensic history}

Fifteen women (17.6\%) reported current charges at the time of initial assessment by the DLM. Twenty-six women $(31.7 \%)$ reported a history of having at least one prior conviction and $18(22.5 \%)$ of them had been in prison at least once.

\subsection{Viral status at presentation}

The majority of women were unaware of their HIV $(60.4 \%)$, HBV $(58 \%)$, or HCV $(56.2 \%)$ status at the time they met the DLM. All were offered viral testing as part of the routine prenatal work-up and the majority agreed to this. Subsequently, six women $(6.3 \%)$ were HIV positive, four women (4.2\%) were HBV positive, and 67 women $(69.8 \%)$ were $\mathrm{HCV}$ positive.

\subsection{Psychiatric co-morbidity}

Data on psychiatric co-morbidity was not routinely collected. Nonetheless, 39 women (42.9\%) reported that they had a past history of depression. None reported a

Table 1

\begin{tabular}{lccc}
\hline & Mean & $\begin{array}{l}\text { Standard } \\
\text { deviation }\end{array}$ & Range \\
\hline $\begin{array}{l}\text { Age } \\
\begin{array}{c}\text { Age leaving education } \\
\text { in years }\end{array}\end{array}$ & 24.07 & 4.51 & $17-38$ \\
$\begin{array}{c}\text { Age at first heroin } \\
\text { use in years }\end{array}$ & 15.74 & 1.37 & $12-18$ \\
$\begin{array}{c}\text { Duration of injecting } \\
\text { heroin use in months }\end{array}$ & 18.33 & 4.13 & $9-32$ \\
$\begin{array}{c}\text { Methadone dose at } \\
\text { presentation in mg }\end{array}$ & 20.61 & 29.49 & $0-108$ \\
$\begin{array}{c}\text { Methadone dose at } \\
\text { delivery in mg }\end{array}$ & 41.65 & 23.54 & $0-90$ \\
\hline
\end{tabular}

history of psychosis. Fourteen women (16.5\%) had had at least one admission to an in-patient psychiatric unit. Seven women $(8.2 \%)$ were attending a General Adult Psychiatry OPD at initial presentation.

\subsection{Stabilization of drug use}

Fifty-three women (53.5\%) were assessed initially as being unstable in terms of their opioid use, 31 women (32.3\%) were offered admission to a specialist inpatient Drug dependency unit to stabilize, and 26 of those women were actually admitted. The mean duration of stay was 4.89 days (range 1-49 days). All returned to outpatient treatment. Twenty-one women $(24.7 \%)$ were assessed as needing stabilization of their benzodiazepine use. Of these 21 women, $17(20.2 \%)$ were admitted to the specialist unit to detoxify from benzodiazepines. Their mean duration of stay was 3.72 days (range 1-44 days). In total, 11 women discontinued their benzodiazepines and ten women achieved a reduction of their prescribed doses.

\subsection{Prenatal care}

Mean gestation at the index appointment in the $\mathrm{CWH}$ was 18.44 weeks (range 7-35). Thirty-one women (45.6\%) were seen at or before sixteen weeks. Prenatal attendance was recorded as being satisfactory (five or more visits) in $56(62.9 \%)$ women, and as being unsatisfactory in 29 (32.6\%) women, using criteria employed by the CWH.

\subsection{Obstetric outcomes}

There were no maternal deaths. Thirteen women (12.7\%) had caesarean sections. The percentage of caesarean sections in our cohort $(12.7 \%)$ is lower than the corresponding percentage for the $\mathrm{CWH}$ in 1999 at $16.3 \%$ of all births (Coombe Women's Hospital, 2000, p. 10).

\subsection{Neonatal outcomes}

The mean gestation at delivery was 38.45 weeks (range 17-43 weeks). The mean birth weight of the babies was $2948.91 \mathrm{~g}$ (range $1130.00-4120.00, S D=608.47$ ). Twelve infants $(10.5 \%)$ were delivered at a gestational age of less than 37 weeks, i.e. premature. This compares with an overall percentage of $5.8 \%$ for babies delivered prematurely in CWH for 1999 (Coombe Women's Hospital, 2000, p. 13) and $5.9 \%$ of all births in 1998 (Coombe Women's Hospital, 1999, p. 13). It is less than previous UK estimates of the prevalence of prematurity among the offspring of pregnant drug dependent women at $20 \%$ to $33 \%$ (Klenka, 1986). This cohort had 2 infants born at less than 28 weeks (1.8\%) and 10 infants born between 28 and 36 weeks (8.8\%). Figures for 1999 from the CWH show $0.3 \%$ of children born at less than 28 weeks gestation and $5.5 \%$ born between 28 and 36 weeks. There was one late neonatal death at 28 days. 
Unfortunately permission for an autopsy was refused and a pathological diagnosis was not established.

\subsection{Special Care Baby Unit admission}

Forty-four babies (42.7\%) of this entire cohort were admitted to the Special Care Baby Unit. Sixteen of these (15.7\%) had a principal diagnosis of Neonatal Abstinence Syndrome (NAS), $13(12.7 \%)$ had a diagnosis of NAS with a co-existing medical problem, 5 (4.9\%) were admitted solely because of problems associated with their premature status, and 9 (8.8\%) had other medical diagnoses. One baby did not have a recorded diagnosis. The mean length of stay was 5.88 days (range $1-117$ days). The latter stay was an extreme outlier for a baby born at 22 weeks gestation.

\subsection{Maternal methadone dose and neonatal withdrawals}

Of this cohort of women, 85 (75\%) were being prescribed methadone at the time of delivery and their infants' withdrawal status was known. We recorded maternal methadone dose at delivery, alcohol and tobacco use status and the presence of positive urinalysis for other opioids, benzodiazepines and cocaine, 1 month pre-delivery, with a view to examining the risk factors for infant withdrawals. Because only three women were non-smokers and three used cocaine, these variables were omitted from the analysis.

We were aware that the treatment received by the mother could influence the likelihood of neonatal withdrawal and therefore recorded a rating of adequacy of maternal attendance at prenatal appointments (as recorded by the obstetric service) and whether the mother had been admitted to a specialist inpatient unit for opioid or benzodiazepine stabilization. Maternal methadone doses, which ranged from 5 to $95 \mathrm{mg}$, were classified into three levels for the analysis, each containing a similar number of women: Low: $n=29$ (5-30 mg), Medium: $n=28(31-50 \mathrm{mg})$ and High: $n=28$ (51-95 mg). Gestation was also classified into three levels: Early (23-37 weeks), On time (38-40 weeks) and Late (41 weeks + ).

Forty infants (47\%) received a diagnosis of Neonatal Abstinence Syndrome using the Finnegan neonatal abstinence scoring system (Finnegan, Connaughton, Kron, \& Emich, 1975). Women whose infants had withdrawals had a median methadone dose at delivery of $52.5 \mathrm{mg}$ (Interquartile range: $=40.0-63.7 \mathrm{mg}$ ) compared to a mean dosage of $34.0 \mathrm{mg}$ (Interquartile range: $=20.0-45.0 \mathrm{mg}$ ) for those methadone maintained women whose babies did not exhibit withdrawal, a highly significant difference (Mann-Whitney $\mathrm{U}=498.5, p<.001)$. The chi-square test for trend indicated that there was a significant dose-response relationship between maternal methadone dose at delivery and the occurrence of withdrawals $\left(\chi^{2}=10.825, d f=1, p=.001\right)$. Respectively, withdrawal occurred in $28 \%$ (8/29), $43 \%$ $(12 / 28)$ and $71 \%(20 / 28)$ of infants delivered to women at low, medium, and high doses ( Table 2).
Table 2

Relationship between methadone dose level and neonatal withdrawal

\begin{tabular}{|c|c|c|c|c|}
\hline \multirow[b]{3}{*}{ Methadone dose at delivery } & \multicolumn{4}{|c|}{ Infant withdrawals } \\
\hline & \multicolumn{2}{|c|}{ Yes } & \multicolumn{2}{|c|}{ No } \\
\hline & $n$ & $\%$ & $n$ & $\%$ \\
\hline Low (5-30 mg) & 8 & $27.6 \%$ & 21 & $72.4 \%$ \\
\hline Medium $(31-50 \mathrm{mg})$ & 12 & $42.9 \%$ & 16 & $57.1 \%$ \\
\hline High $(51-95 \mathrm{mg})$ & 20 & $71.4 \%$ & 8 & $28.6 \%$ \\
\hline All & 40 & $47.1 \%$ & 45 & $52.9 \%$ \\
\hline
\end{tabular}

Withdrawals occurred in, respectively, 29\% (5/17), 49\% $(21 / 43)$ and $52 \%(11 / 21)$ of infants delivered early $(<38$ weeks), on time (38-40 weeks) and late ( $>40$ weeks). However, this graded relationship was not statistically significant. The presence of opioids other than methadone in the month pre-delivery and the mother's alcohol use status were not related to the occurrence of infant withdrawal. However, a significant association was found between infant withdrawal and mother's benzodiazepine use $\left(\chi^{2}=4.377, d f=1, p<.05\right)$. Withdrawals occurred in $59 \%$ (20/34) of cases where the mother tested benzodiazepinepositive in the month preceding delivery compared to $33 \%$ (11/33) of cases where the mother tested negative.

None of the associations between frequency of withdrawal and adequacy of prenatal appointment attendance, admission for benzodiazepine stabilization or for opioid stabilization was statistically significant.

Multivariate logistic regression (Table 3) indicated that benzodiazepine use was not independently associated with withdrawal (adjusted OR $=1.704$, 95\% CI: 0.554-5.238), whereas high maternal methadone dose at delivery remained a significant risk factor (adjusted OR $=4.862,95 \%$ CI: 1.166-20.273).

\subsection{Discharge home and neonatal followup}

All infants were discharged in the custody of their birth mothers. Only 11 (11.5\%) women returned with their babies for a developmental check up at 4 months. Limited information was available on the prevalence of neurodevelopmental problems in this group. Of the 11 babies who returned to the Developmental Clinic, six were noted to have developmental problems and four of these were recorded as central nervous system-related.

\section{Discussion}

This paper extends our knowledge of pregnant opioid dependent women by describing a sample who received prenatal care from a specialized Drug Liaison Midwife service associated with the Coombe Women's Hospital in Dublin, Ireland. Though purely descriptive at this point, the findings suggest benefits associated with this service model and helps establish that effective working relationships are 
Table 3

Results of separate and multivariate logistic regression analyses to assess the risk of withdrawal

\begin{tabular}{|c|c|c|c|c|}
\hline Variable & Crude OR & $95 \% \mathrm{CI}$ & Adjusted OR & $95 \% \mathrm{CI}$ \\
\hline Maternal methadone dose (ref. Low: $5-30 \mathrm{mg}$ ) & 1.000 & & 1.000 & \\
\hline Medium: $31-50 \mathrm{mg}$ & 1.969 & $0.651-5.950$ & 2.401 & $0.633-9.101$ \\
\hline High: $51-95 \mathrm{mg}$ & 6.563 & $2.066-20.842$ & 4.862 & $1.166-20.273$ \\
\hline Gestation (ref. Early: <38wks) & 1.000 & & & \\
\hline On time: $38-40 \mathrm{wks}$ & 2.290 & $0.688-7.620$ & & \\
\hline Late: 41 wks + & 2.639 & $0.684-10.175$ & & \\
\hline Antenatal appt. attendance (ref. Average/above average) & 1.000 & & & \\
\hline Below average & 1.558 & $0.579-4.195$ & & \\
\hline Alcohol (ref. abstainers) & 1.000 & & & \\
\hline Alcohol drinker & 0.867 & $0.339-2.219$ & & \\
\hline Benzodiazepines* (ref. tested negative) & 1.000 & & 1.000 & \\
\hline Tested positive & 2.857 & $1.056-7.727$ & 1.704 & $0.554-5.238$ \\
\hline Opioids* (ref. tested negative) & 1.000 & & & \\
\hline Tested positive & 1.448 & $0.572-3.665$ & & \\
\hline Admission for benzodiazepine stabilisation (ref. No) & 1.000 & & & \\
\hline Yes & 1.929 & $0.653-5.692$ & & \\
\hline Admission for opioid stabilisation (ref. No) & 1.000 & & & \\
\hline Yes & 0.485 & $0.133-1.767$ & & \\
\hline
\end{tabular}

* Tested one month pre-delivery.

possible between pregnant opioid dependent women and the Obstetric and Drug Services involved in their care. The analysis also suggests a positive association between magnitude of maternal methadone dose and likelihood of neonatal withdrawal.

Substance users are universally unpopular with the public and even with health professionals. There is a substantial literature on the negative perceptions about women who are drug dependent and in particular those who are dependent and pregnant. Stigma is a reality for many of these women and impedes their appropriate care (Crisp, Gelder, Rix, Meltzer, \& Rowlands, 2000). It was envisaged that the creation of a specialist Drug Liaison Midwife would assist in the reduction of the stigma associated with the treatment of pregnant female drug users, ease access to care for pregnant dependent women and help maintain care pathways once established (Scully, Geoghegan, \& Keenan, 2001). It was also envisaged that the post would facilitate better communication and working relationships among the professional staff involved in the care of this patient population. The experience of these DLMs suggests that this is a feasible and attainable aim.

Most of the women were enlisted in the antenatal care through their involvement with the Drug Treatment services. All but one of the women were retained in Drug treatment throughout the course of their pregnancies. The majority of the women stabilized on methadone. A minority however remained quite difficult to stabilize on an outpatient basis. Admission to a specialist drug dependency unit helped many of them but a minority (35 women $(36.1 \%)$ in the month pre-delivery and 17 women $(18.7 \%)$ in the week predelivery) remained opioid positive.

The obstetric and neonatal outcomes appear generally in line with other European/UK studies where a dedicated service is provided for pregnant opioid dependent women
(Myles, 2000) and significantly better than in studies of cohorts of pregnant drug dependent women where no specialist service exists. The level of prematurity $(10.5 \%$ with a gestational age below 37 weeks) is lower than the range (20-33\%) commonly quoted in the literature (Klenka, 1986), but obviously remains of concern.

Although the case for methadone maintenance in pregnancy is now well established (Ward, Hall, \& Mattick, 1999) and it is accepted that for the majority of opioid dependent pregnant women (Fisher, 2000), there exist continuing concerns about the relationship between maternal methadone dose and the subsequent development of neonatal withdrawal.

Unfortunately, there is no agreement in the professional literature in respect of the putative association between maternal methadone dose and neonatal withdrawals. The area is controversial and tends to be viewed through the prism of the "maintenance vs. abstinence debate" in respect of substitution therapy (Jarvis \& Schnoll, 1995). Jarvis and Schnoll reviewed the available evidence in a National Institute on Drug Abuse monograph. They noted that a number of North American, Australian, and European studies supported the positive association between methadone dose and likelihood of neonatal withdrawal. They cited studies by Davis, Chappel, Mejia-Zelaya, and Madden in 1975 from the Illinois Drug Abuse Programme, Madden and colleagues in 1977 ( $n=110$ women), and Doberzak, Kandall, and Friedman in $1993(n=21)$ that supported the contention that a positive relationship exists. A UK study by Dawe, Gerada, and Strang $(\mathrm{n}=35)$ published in 1992 also noted a positive correlation between maternal methadone dose and neonatal withdrawals.

Studies with negative findings were also noted in the Jarvis and Schnoll's NIDA review (1995): Harper, Solish, Purow, Sang, and Panepinto (1974) in New York did not 
find evidence of any positive correlation between maternal methadone dose and neonatal withdrawal. Nor did a later study by Kandall and colleagues (1977). Subsequent studies by Brown et al. (1998) and Berghella et al. (2000) did not find evidence of a positive correlation between maternal methadone dose and neonatal withdrawals. Berghella and colleagues noted that in a retrospective review of 51 pregnant opioid dependent women attending their institution between 9/96 and 9/97 with a mean maternal methadone dose of $83.4 \mathrm{mg}$ (range $30-165 \mathrm{mg}$ ) before delivery that high maternal methadone doses might not significantly worsen neonatal withdrawal compared to lower doses.

More recently a paper by Dashe and colleagues (2002) described a retrospective cohort study of 70 pregnant opioid dependent women attending their facility in Texas, USA. They described a significant relationship between maternal methadone dose and neonatal withdrawal. In a UK setting a paper by Johnson, Greenough, and Gerada (2003) examining 41 admissions to a tertiary neonatal intensive care unit in London demonstrated that mothers who took methadone and other illicit substances in pregnancy had significantly longer neonatal admissions than infants of women with other types of drug use.

It is apparent that the field is divided on the issue of a relationship and the significance to attach to this relationship, should it be demonstrated. Every decision to prescribe any pharmacologically active agent represents the end point of a process of decision making where the potential risks and benefits in addition to the patient's views on the matter are weighed, considered, and a recommendation made. Neonatal withdrawals are a treatable complication of oral substitution therapy, whereas prematurity, low birth weight and seroconversion for either HCV or HIV have much more serious implications for the infant's future health.

\section{Limitations}

While this is one of the largest series reported to date in the literature, involving a single Dublin maternity hospital, there are a number of limitations to this analysis. First, the analyses are subject to the methodological shortcomings associated with a retrospective chart based survey. The survey instrument was developed locally and is not a standardized instrument. Many variables in the dataset were missing, also reducing the specificity of our findings. Several variables not measured or analyzed may have acted as confounding factors (e.g. maternal smoking status, as all but three women smoked) and we were not in a position to control for these. Self selection by our patients, may have biased the results of the analysis. That is, these findings may not extend to pregnant women dependent upon other substances, or to pregnant opiate dependent women who did not seek pre-natal care.

As noted care was tailored individually according to perceived need and individual choice, methadone doses were decided collaboratively by the prescribing doctor and the woman concerned on the basis of self reported withdrawals, observed withdrawal symptoms, and the results of regular urine toxicology. Finally the ethos of the service was to encourage stabilization of drug use and engagement with pre-natal and social care. There was a possibility of bias in the formulation of a diagnosis of neonatal withdrawals on the part of the treating clinicians; this was limited by the regular use of a validated instrument in making the diagnosis of neonatal withdrawals.

In summary, the experience of this specialized Drug Liaison Midwife liaison service indicates that it is possible to build effective working relationships between drug dependent pregnant women, the obstetric services, and the drug treatment services involved in their care. The obstetric and neonatal outcomes of this population appear to have improved with the introduction of this specialized service with benefit to these mothers, their infants and to the health care system - although this suggestion remains tentative since our study did not involve a control group of similar patients who did not receive the DLM service. We believe that our experience with Drug Liaison Midwife service adds support for a policy of developing similar liaison posts in other drug treatment services where there are significant numbers of pregnant drug-using women.

\section{Acknowledgments}

The authors would like to thank the South Western Area Health Board for funding. They also wish to thank the Director of Nursing, Ms. Ita Dwyer, and Drs. Sean Daly, Michael Turner, and Christopher Fitzpatrick of the Obstetrics department, Drs. Martin White and Margaret BurkeSheridan of the Neonatology department, and the medical records staff in both departments for their assistance.

This study was funded by the South Western Area Health Board of the Eastern Regional Health Authority, Ireland.

\section{References}

Barry, J. (2002). Policy response to opioid misuse in Dublin. Journal of Epidemiology and Community Health, 56, 7-8.

Berghella, V., Lim, P., Cherpes, J., Hill, M. K., Kaltenbach, K., \& Wapner, R. J. (2000). Maternal methadone dose and neonatal withdrawal. American Journal of Obstetrics and Gynecology, 182, S154 (abstract).

Brown, H. L., Britton, K. A., Mahaffey, D., Brizendine, E., Hiett, A. K., \& Turnquest, M. A. (1998). Methadone maintenance in pregnancy: A reappraisal. American Journal of Obstetrics and Gynecology l, 179, $459-463$.

Coombe Women's Hospital. (1999). Annual Clinical Report 1998. Dublin, Ireland: Coombe Women's Hospital.

Coombe Women's Hospital. (2000). Annual Clinical Report 1999. Dublin, Ireland: Coombe Women's Hospital.

Crisp, A. H., Gelder, M. G., Rix, S., Meltzer, H. I., \& Rowlands, O. J. (2000). Stigmatisation of people with mental illnesses. British Journal of Psychiatry, 177, 4-7. 
Dashe, J. S., Sheffield, J. S., Olscher, D. A., Todd, S. J., Jackson, G. L., \& Wendel, G. D. (2002). Relationship between Maternal Methadone Dosage and Neonatal Withdrawal. Obstetrics and Gynecology, 100, $1244-1249$.

Davis, R. C., Chappel, J. N., Mejia-Zelaya, A., \& Madden, J. (1975). Clinical observations on methadone maintained pregnancies. Journal of Addictive Diseases, 2, 101-112.

Dawe, S., Gerada, C., \& Strang, J. (1992). Establishment of a liaison service for pregnant opioid dependent women. British Journal of Addiction, 87, 867-871

Department of Tourism, Sport \& Recreation. (2001). Building on Experience. National Drugs Strategy 2001-2008. Section 2.4, National and Regional Trends (pp. 23-24). Dublin, Ireland: Author.

Doberzak, T. M., Kandall, S. R., \& Friedman, P. (1993). Relationship between maternal methadone dosage and maternal-neonatal methadone levels, and neonatal withdrawals. Obstetrics and Gynecology, 81, 936-940.

Ebrahim, S. H., \& Gfroerer, J. (2003). Pregnancy related substance use in the United States during 1996-1998. Obstetrics and Gynecology, 101, $374-379$.

European Monitoring Centre for Drugs and Drug Addiction. (2000). Annual report on the state of the drug problem in the European Union. Luxemburg: Office for Official Publications of the European Communities.

Finnegan, L. P., Connaughton, J. F., Kron, R. E., \& Emich, J. P. (1975). Neonatal Abstinence Syndrome: Assessment and management. Journal of Addictive Diseases, 2 (2), 141-158.

Fischer, G. (2000). Treatment of opioid dependence in pregnant women. Addiction, 95, 1141-1144.

Harper, R. G., Solish, G. I., Purow, H. M., Sang, E., \& Panepinto, W. C. (1974). The effect of a methadone treatment program upon pregnant heroin addicts and their newborn infants. Pediatrics, 54, 300-305.

Jarvis, M. A. E., \& Schnoll, S. H. (1995). Methadone use during pregnancy.
In: C. N. Chiang, \& L. P. Finnegan (Eds.), Medications development for the treatment of pregnant addicts and their infants (pp. 58-75) (National Institute on Drug Abuse Research Monograph Series 149).

Johnson, K., Greenough, A., \& Gerada, C. (2003). Maternal drug use and neonatal unit stay. Addiction, 98, 785-789.

Kandall, S. R., Albin, S., Gartner, L. M., Lee, K., Eidelman, A., \& Lowinson, J. (1977). The narcotic dependent mother: Fetal and neonatal consequences. Early Human Development, 1, 159-180.

Keenan, E., \& Barry, J. (1999). Republic of Ireland has set up scheme to regulate methadone prescribing by GPs. British Medical Journal, $319,1497$.

Klenka, H. M. (1986). Babies born in a district general hospital to mothers taking heroin. British Medical Journal, 293, 745-746.

Madden, J. D., Chappel, J. N., Zuspan, F., Gumpel, J., Mejia, A., \& Davis, R. (1977). Observation and treatment of neonatal narcotic withdrawal. American Journal of Obstetrics and Gynecology, 127, 199-201.

Moran, R., O'Brien, M., \& Duff, P. (1997). National Drug Treatment Reporting System. National Report 1996. Dublin: The Health Research Board.

Myles, J. (2000). Influence of illicit and licit substances for the pregnant drug user and the neonate. Pregnancy and Drug Misuse Update 2000, a seminar organised by the Co-operation Group to Combat Drug Abuse and Illicit Trafficking in Drugs (Pompidou Group) (pp. 21-29). Strasburg: Council of Europe Publishing.

O'Brien, M., Moran, R., Kelleher, T., \& Cahill, P. (2000). National Drug Treatment Reporting System, Statistical Bulletin, 1997 and 1998. National Data and Data by Health Board Area. Dublin: The Health Research Board.

Scully, M., Geoghegan, N., \& Keenan, E. (2001). Drug liaison midwives. Addiction, 96, 651-652.

Ward, J., Hall, W., \& Mattick, R. P. (1999). Role of maintenance treatment in opioid dependence. Lancet, 353, 221-226. 